\title{
A EXTENSÃO UNIVERSITÁRIA NO FORTALECIMENTO DA REDE DE CUIDADOS À PESSOA COM DEFICIÊNCIA
}

\author{
THE UNIVERSITY EXTENSION IN \\ STRENGTHENING THE CARE NETWORK FOR \\ PEOPLE WITH DISABILITIES
}

\author{
Ana Carolina Steiner Stüpp ${ }^{1}$ \\ Adrielly Marconato Durante ${ }^{2}$ \\ Ana Paula Marcelino de Aquino ${ }^{3}$ \\ Camila Carolain da Cruz Semioni ${ }^{4}$ \\ Graciele Pêssoa e Silva ${ }^{5}$ \\ Leonardo Perão ${ }^{6}$ \\ Indiara de Mesquita Fialho ${ }^{7}$ \\ Débora Frizzo Pagnossim ${ }^{8}$ \\ Simone Beatriz Pedrozo Viana ${ }^{9}$
}

RESUMO: O presente estudo tem por objetivo relatar a experiência de um projeto de extensão universitária que busca fortalecer a rede de cuidados à pessoa com deficiência auditiva, física e intelectual, a partir de ações de educação permanente. Este estudo é documental, elaborado a partir da análise qualitativa de conteúdo dos registros das atividades e dos relatórios produzidos pelo projeto de extensão Grupo de Apoio à Rede de Cuidados à Pessoa com Deficiência, nos quais constam as falas dos participantes das capacitações. A experiência vem ampliando a integração ensinoserviço, assim como o vínculo entre os diferentes níveis de atenção à saúde, apoiada na construção de redes de apoio à pessoa com deficiência. Acredita-se que a educação permanente é o caminho para a consolidação e a qualificação do processo de cuidado.

PALAVRAS-CHAVE: Assistência à saúde; Pessoas com deficiência; Profissionais da Saúde.

ABSTRACT: This study reports on the experience of a university extension project aimed at strengthening the care network for people with hearing, physical and intellectual disabilities, based on actions of continuing education. This study is a

Licença CC BY: Artigo distribuído sob os termos Creative Commons, permite uso e distribuição irrestrita em qualquer meio desde que $o$ autor credite a fonte original.

1 Acadêmica do Curso de Fonoaudiologia da Universidade do Vale do Itajaí UNIVALI.

2 Acadêmica do Curso de Psicologia da Universidade do Vale do Itajaí - UNIVALI.

3 Acadêmica do Curso de Fisioterapia da Universidade do Vale do Itajaí - UNIVALI.

4 Acadêmica do Curso de Fonoaudiologia da Universidade do Vale do Itajaí UNIVALI.

5 Acadêmica do Curso de Fonoaudiologia da Universidade do Vale do Itajaí UNIVALI.

6 Acadêmico do Curso de Fisioterapia da Universidade do Vale do Itajaí - UNIVALI.

7 Fonoaudióloga. Mestre em Saúde Pública pela Universidade de São Paulo - USP. Professora titular da UNIVALI.E-mail: indiara@univali.br.

8 Fonoaudióloga. Mestre em Distúrbios da Comunicação Humana pela Universidade Federal de Santa Maria - UFSM. Professora titular da UNIVALI. E-mail: dfrizzo@, univali.br.

9 Fisioterapeuta. Mestre em Saúde e Gestão do Trabalho pela Universidade do Vale do Itajaí - UNIVALI. Professora titular da UNIVALI. E-mail: sviana@univali.br. 
documentary, elaborated based on a qualitative analysis of the contents of the records of activities and reports produced by the extension project "Grupo de Apoio a Rede de Cuidados à Pessoa com Deficiência" (Support Group for the Care Network for People with Disabilities), which includes the discourse of the training participants. This experience helped to extend the integration between teaching and services, and strengthen the bond between the different levels of healthcare, based on the construction of support networks for people with disabilities. It is believed that continuing education is a means of consolidating and qualifying the care process.

KEYWORDS: Healthcare; People with disabilities; Health Professionals.

\section{INTRODUÇÃO}

O censo realizado em 2010 pelo Instituto Brasileiro de Geografia Estatística (IBGE, 2012) aponta que 45,6 milhões de pessoas declaram possuir alguma deficiência, o que representa 23,91\% da população brasileira. A maior parte delas vive em áreas urbanas - 38,5 milhões, sendo a deficiência visual a de maior ocorrência (18,8\%), seguida da física ( $7 \%)$, auditiva $(5,1 \%)$ e intelectual $(1,4 \%)$.

A legislação brasileira considera deficiente toda pessoa que possui limitação ou incapacidade para o desempenho de determinada atividade e se enquadrar nas categorias de: deficiência física, auditiva, visual e/ou intelectual (BRASIL, 2004).

Das pessoas com deficiência, 3,2 \% vivem na região sul do Brasil e, destes, 28,64\% residem em Santa Catarina. No município no qual foram realizadas as oficinas de capacitação, 24,47\% se declaram com algum tipo de deficiência, sendo 14,44\% de deficiências visuais, $5 \%$ de física, 4,2 1\% de auditivas e $0,77 \%$ de intelectual (IBGE, 2012).

Diante deste contexto, o Governo Federal vem ampliando as políticas públicas que visam melhorar o acesso aos bens e serviços disponíveis para toda a população, eliminar barreiras e qualificar $\mathrm{o}$ atendimento às pessoas com deficiência no SUS. Como exemplo destas iniciativas, foi implantado o Plano Nacional dos Direitos da Pessoa com Deficiência - Viver sem Limite, cujo compromisso é oportunizar plena cidadania às pessoas com deficiência no Brasil, a partir de quatro grandes eixos: acesso à educação; inclusão social; atenção à saúde e à acessibilidade. Cada ação presente nesses eixos é interdependente e articulada com as demais, construindo redes de serviços e políticas públicas capazes de assegurar um contexto de garantia de direitos para as pessoas com deficiência, considerando suas múltiplas necessidades nos diferentes momentos de suas vidas (BRASIL, 2015).

Integrando esta rede, foi lançado, em 2012, a Rede de Cuidados à Pessoa com Deficiência, estabelecida pela Portaria Ministerial nº793/12 (BRASIL, 2014), na qual há destaque para qualificação da atenção à saúde por meio da criação, da ampliação e da articulação de pontos de atenção à saúde para pessoas com deficiência temporária ou permanente; progressiva, regressiva ou estável; intermitente ou contínua, no âmbito do Sistema Único de Saúde (SUS). A Rede de Cuidados à Pessoa com Deficiência prevê uma série de estratégias e serviços de atendimento às necessidades de pessoas com deficiência.

Com isso, o Ministério da Saúde propôs a criação dos Centros Especializados em Reabilitação (CER), que visam ao atendimento integral à pessoa com deficiência. Para que esse atendimento ocorra, os serviços de saúde devem estruturar uma rede de atenção à pessoa com deficiência que contemple ações de promoção, proteção, recuperação e reabilitação, respeitando o princípio doutrinário da integralidade. 
A Universidade do Vale do Itajaí(UNIVALI), desde 2005, credenciou ao Ministério da Saúde o Serviço Ambulatorial de Saúde Auditiva (SASA), que tem por objetivo prestar assistência especializada às pessoas com deficiência auditiva para 53 municípios de Santa Catarina, nos níveis de média e alta complexidade e, em 2013, credenciou o Centro Especializado em Reabilitação II (CER II - deficiência física e intelectual), a fim de ampliar o atendimento à pessoa com deficiência física e intelectual.

O SASA tem por finalidade prestar assistência especializada às pessoas com doenças otológicas, especialmente para aquelas com deficiência auditiva. Este serviço realiza desde a triagem e o monitoramento auditivo até a reabilitação do usuário de AASI (SILVA; GONÇALVES; SOARES, 2014).

O CER, na área da deficiência física, oferece serviços de reabilitação, tais como: protetização a amputados; atenção, dispensação de equipamentos coletores e orientação ao autocuidado aos ostomizados; reabilitação decorrente de paralisia cerebral, tetraplegia, hemiplegia, paraplegia e nanismo (PEREIRA; MACHADO, 2016). Na reabilitação para pessoas com deficiência intelectual e/ou Transtornos do Espectro do Autismo, o CER garante cuidados em saúde, visando ao desenvolvimento de habilidades singulares no âmbito do projeto terapêutico, voltadas à cognição, à linguagem e à socialização.

Pelo fato do CER II ter uma implantação recente, existe ainda pouca articulação deste com outros serviços e o fluxo se resume aos encaminhamentos recebidos de outros locais de atenção à saúde. Além disso, observa-se que os municípios não possuem estrutura de atendimento compatível com a necessidade desta população.

Sendo assim, para efetivação destes serviços, surge a necessidade da qualificação desta rede de cuidados. A UNIVALI, por ser uma universidade comunitária, cuja missão é produzir e socializar o conhecimento pelo ensino, pesquisa e extensão, estabelecendo parcerias solidárias com a comunidade, sente-se no compromisso de desempenhar um papel social e, nesse sentido, auxiliar na construção e na qualificação desta rede. Para tal, utiliza-se o conceito de extensão universitária como meio de interação com a sociedade, cujo objetivo é produzir, ensinar e aplicar o conhecimento (AYRES, 2015).

A partir desta lógica e considerando as inúmeras solicitações advindas da comunidade, nasceu o projeto de extensão: Grupo de Apoio à Rede de Cuidados à Pessoa com Deficiência, visando fortalecer e qualificar a rede de cuidados nos municípios da Associação dos Municípios da Foz do Rio Itajaí (AMFRI).

A proposta do projeto está alicerçada na educação permanente e leva em consideração os conhecimentos preexistentes dos profissionais da Atenção Básica. Fazem parte do projeto professores e acadêmicos do curso de fonoaudiologia, fisioterapia e psicologia, abrangendo as deficiências auditiva, física e intelectual.

Informações extraídas da literatura afirmam que se precisa avançar nessa temática, por meio da qualificação das ações implantadas, visto que a deficiência ainda está ancorada no modelo biomédico e o desenho atual da Rede de Cuidados à Pessoa com Deficiência possui barreiras de acesso à saúde das pessoas com deficiência (URSINE; PEREIRA; CARNEIRO, 2018)

Assim, o objetivo do presente artigo é relatar a experiência de um projeto de extensão universitária que busca fortalecer a rede de cuidados à pessoa com deficiência auditiva, física e intelectual, a partir de ações de educação permanente. 


\section{METODOLOGIA}

Trata-se de um estudo documental, elaborado a partir da análise de conteúdo dos registros das atividades e dos relatórios produzidos pelo projeto de extensão Grupo de Apoio à Rede de Cuidados à Pessoa com Deficiência, nos quais constam falas dos participantes das capacitações. Os resultados são apresentados de forma descritiva, com inclusão de recortes de falas que tiveram maior ocorrência durante as capacitações, identificadas pela letra $\mathrm{P}$, seguida de um número, a fim de respeitar os preceitos éticos em pesquisa, mantendo o anonimato dos profissionais que frequentaram as capacitações.

Participaram das capacitações agentes comunitários de saúde (ACS); técnicos em enfermagem; enfermeiros; médicos; auxiliares administrativos e a fonoaudióloga gestora, totalizando 120 profissionais.

O planejamento das capacitações iniciou pelo levantamento da demanda junto à gestão de saúde do município, ou seja, foram verificados os assuntos de interesse e as dificuldades enfrentadas pelo grupo de profissionais no que se refere à organização e à compreensão do fluxo de encaminhamento específico para o CER II e SASA.

Estabelecidos os fluxos, foi elaborada uma agenda de capacitações, contendo os temas que seriam abordados. As capacitações foram previamente planejadas com a gestora de saúde, sendo o cronograma elaborado de acordo com a disponibilidade de cada Unidade Básica de Saúde (UBS). Ao todo foram realizadas oito capacitações.

A coordenação das capacitações foi realizada pelos membros do projeto de extensão, estando presentes um professor e dois acadêmicos, sendo um do curso de fisioterapia e um do curso de fonoaudiologia e, eventualmente, um acadêmico do curso de psicologia.

As capacitações tinham como tema principal os serviços CER II e SASA, e incluíam: apresentação; áreas de abrangência; distribuição de vagas; perfil dos usuários; deficiências atendidas; fluxo externo de encaminhamentos específico do município; fluxos internos dos serviços; composição das equipes multidisciplinares; atividades executadas; estratégias para acompanhamento dos usuários, incluindo o sistema de contrarreferência e os grupos de apoio existentes; principais dificuldades encontradas; critérios de indicação para aparelho de amplificação sonora individual (AASI) no SASA; e uma breve explicação do projeto de extensão e de deficiência. Além disso, ao final das capacitações, foram realizados estudos de caso de usuários específicos de cada UBS e esclarecidas dúvidas pontuais.

A metodologia utilizada para a realização das capacitações envolveu recursos audiovisuais, rodas de conversa e estudos de caso.

O tempo médio das capacitações foi de três horas e a avaliação foi realizada com a aplicação de uma ficha elaborada para este fim com três colunas: (1) que bom - relato dos pontos positivos da atividade; (2) que pena - relato dos pontos negativos; e (3) que tal - sugestões para as próximas capacitações. Além deste instrumento, os participantes preenchiam outra ficha em que indicavam temas para novas capacitações. 


\section{RESULTADOS E DISCUSSÕES}

Durante as capacitações, o tema principal abordado foi o funcionamento do SASA e do CER II, e a articulação destes com a atenção básica, bem como o fluxo que os usuários seguem para atendimento nos serviços.

Considerando a potencialidade da metodologia dialogada e participativa, os profissionais tinham a liberdade de realizar questionamentos e compartilharem casos atendidos, a fim de exemplificar o que era explicado e dar resolubilidade aos casos.

A gestão participativa é um modo de mobilizar o trabalhador, o que inclui o pensar e o fazer coletivo. Deve-se conhecer a realidade institucional, criar espaços de discussões para contextualizar impasses e pensar e decidir coletivamente. A educação permanente é baseada na aprendizagem significativa, construindo saberes a partir de experiências das pessoas por meio de rodas de conversa, oficinas e reuniões que propõem soluções e mudanças (TOCCI; COSTA, 2014).

Todo o profissional da saúde é operador de cuidados no âmbito indivíduo, família, comunidade e nas tecnologias leves do cuidado, entendidas como as existentes nas relações e na produção da comunicação, do acolhimento, do vínculo e da autonomização. Para tanto, os profissionais deverão ser capacitados para a qualificação e o fortalecimento da Atenção Básica em Saúde (SANTOS; MIRANDA, 2016).

As demandas para capacitação não se definem somente a partir de uma lista de necessidades individuais de atualização, mas, prioritariamente, a partir das problematizações que acontecem no dia a dia do trabalho referentes à atenção à saúde e à organização do trabalho, garantindo uma aplicabilidade e relevância dos conteúdos e das tecnologias estabelecidas (ALVES, 2017).

As capacitações tiveram também o objetivo de instrumentalizar os profissionais da saúde quanto à identificação, ao registro, ao encaminhamento e ao acompanhamento de usuários com deficiência auditiva, física ou intelectual, bem como preparar multiplicadores no município. Procurou-se também discutir o funcionamento dos serviços e buscar técnicas que facilitassem a comunicação entre a atenção básica e os serviços especializados.

Foram apresentadas outras opções de interação, gerenciadas pelo projeto de extensão, com o conhecimento repassado nas capacitações, como o Telessaúde, que consiste em uma ferramenta de consultoria on-line, utilizado para solucionar questionamentos e situações vindas dos municípios de abrangência do SASA desde novembro de 2016 e os blogs dos dois serviços, os quais são atualizados rotineiramente com informações diversas sobre estes, além de um $e$-mail que objetiva responder dúvidas referentes aos serviços.

Dentro do novo contexto criado pela globalização, a tecnologia de informação fornece possibilidades de permanente atualização e integração do conhecimento, visto que potencializa os processos de tratamento, disseminação e transferência de informações (FERREIRA; CURRY, 2013).

A avaliação das capacitações revelou uma boa aceitação pelos profissionais, uma vez que houve maior expressão de comentários positivos ("que bom”) do que negativos ("que pena”). As respostas com maior ocorrência serão apresentadas por meio de recortes de fala ao longo dos resultados e das discussões do presente artigo.

No aspecto positivo ("que bom”), houve dois grandes grupos de qualificação, sendo o primeiro relacionado à apreciação da exposição do tema: 
"Gostei da palestra, muito educativa e produtiva." (P1).

"Existe este trabalho maravilhoso e que hoje conseguimos conhecer melhor" (P2)

"Muito bom, achei muito interessantes a palestra" (P3)

"Esclareceu vários temas que nós não tínhamos ideia do que se tratava" (P4)

"Gostei de saber sobre as deficiências e como funciona" (P5)

O segundo grupo de respostas está relacionado às colaborações na melhora da resolutividade, considerando a intencionalidade das oficinas em si. Neste sentido, o conteúdo das falas, a seguir exemplificado, expressa satisfação:

"Conhecer um pouco sobre os serviços oferecidos, fluxos e visão geral da equipe multiprofissional envolvida (...)” (P6)

"Ajudará bastante com a orientação dos pacientes" (P7)

"Entender o fluxo de encaminhamento e conhecer os serviços realizados" (P8)

"Foi muito útil para nossa rotina" (P9)

Entre os aspectos negativos (“que pena”), foram observados quatro grupos de respostas, sendo o primeiro relacionado à frequência e ao tempo das capacitações:

"Que o tempo foi pouco!" (P1O)

"Que faltou mais tempo." (P11)

"Que foi um encontro" (P12)

"Não ser algo mais completo" (P6)

Também foram considerados aspectos negativos em relação à rede de cuidados à pessoa com deficiência:

"O sistema não consegue abranger todas as necessidades e todos os que precisam" (P13)

"Não termos mais centro de reabilitação" (P14)

"Que muitos não têm acompanhamento" (P15)

No terceiro grupo, observa-se um desejo por parte dos participantes de que mais pessoas tivessem acesso à capacitação:

"Que não houve participação de alguém do PAI e NAI aqui com nós" (P16)

"Outros profissionais não estarem presentes" (P17)

Ainda, em um quarto grupo, reuniram-se as respostas que demandavam mais conhecimento prático sobre o assunto:

"Não terem trazido os aparelhos auditivos" (P8)

"Poderia ter tido mais fotos e depoimentos" (P5)

"Não poder conhecer o ambiente" (P18)

No item de avaliação de sugestões (“que tal”), as respostas foram divididas em três grupos, no primeiro os profissionais citam a importância de materiais de apoio sobre os temas abordados:

"Algum material de apoio para servir como informativos para as pessoas portadoras e seus familiares" (P6) 


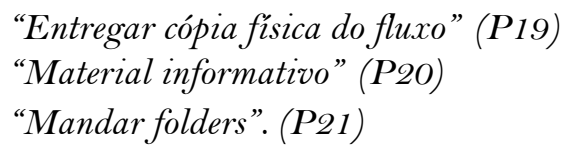

Nesta categoria, no grupo 2, novamente houve a demanda de capacitações com mais frequência abordando mais temas:

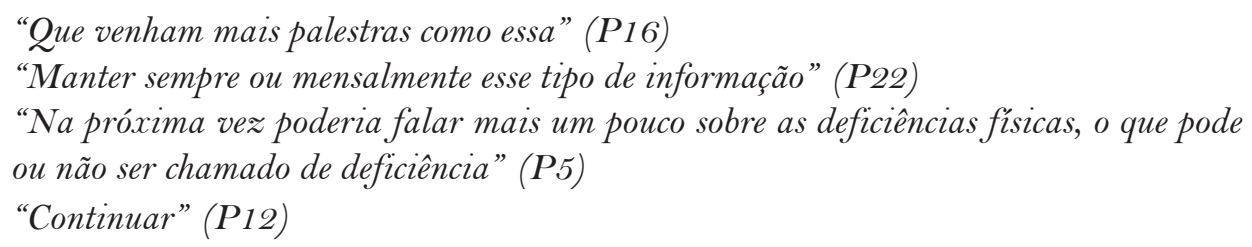

Em uma última categoria, foram agrupadas sugestões que novamente demandavam maior conhecimento prático:

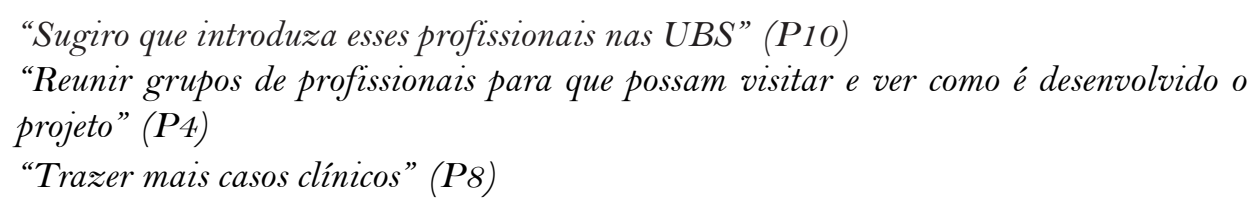

As avaliações realizadas mostraram que os assuntos abordados foram de interesse dos profissionais. Diante das numerosas e diversas sugestões, o projeto de extensão criou um folder informativo contendo um "Menu de Capacitações", no qual constam propostas de formação para que sejam escolhidas pelas UBS para continuidade das capacitações no ano de 2017.

Os relatos positivos dos participantes estão de acordo com Godoy, Guimarães e Assis (2014), que em estudo com o objetivo de avaliar um programa de capacitações para a atenção básica, apontaram que o profissional deste nível busca alternativas de capacitação para garantir assistência de qualidade e qualificação profissional, uma vez que as demandas de atenção à saúde requerida pela comunidade por vezes apresentam-se complexas e de difícil resolutividade no nível local.

Ainda, com base nas sugestões, foram elaboradas cartilhas informativas e cartazes sobre os temas das capacitações do SASA e do CER II, que serão entregues em todas as UBS participantes, tão breve estejam publicadas. Além disso, mais capacitações foram agendadas para o ano subsequente em outros municípios de abrangência dos serviços.

A Educação Permanente em Saúde (EPS) faz parte de um processo de reestruturação dos serviços diante de novas demandas, favorecendo a articulação dos princípios epistemológicos, científicos, estéticos e tecnológicos, na busca da melhoria na qualidade de vida para usuários e profissionais (LEMOS, 2016).

A EPS envolve processos políticos, sociais, econômicos, desejos e demandas pessoais, pensamentos ideológicos, diferenças disciplinares profissionais e instituições formadoras e são permeadas por dificuldades de infraestrutura material, de gestão e de recursos humanos para desenvolver ou continuar multiplicando e aplicando a educação permanente (MICCAS; BATISTA, 2014).

Assim, implementar processos de ensino aprendizagem que sejam respaldados por ações crítico-reflexivas e participativas de que a promover mudanças nas diferentes realidades de cada serviço é o principal desafio para atingir as metas propostas pelos documentos da OPAS/OMS e Ministério da Saúde (MICCAS; BATISTA, 2014). 


\section{CONSIDERAÇÕES FINAIS}

As oficinas de capacitações, realizadas pelo projeto de extensão do ano de 2016 nas UBS de um município do litoral catarinense cumpriram o papel da extensão universitária, entendendo que este se fundamenta no benefício da comunidade, mesmo que de forma indireta. A capacitação da equipe da ESF acerca dos serviços que compõem a rede de cuidados à pessoa com deficiência certamente originará reflexos positivos à comunidade dos respectivos territórios, visto que passarão a ter maior facilidade de acesso a esses serviços, antes, por vezes, desconhecidos.

O depoimento dos profissionais, alvo das capacitações, reafirma a importância da temática escolhida, ao mesmo tempo em que confirmou lacunas existentes em relação aos fluxos de encaminhamento, assim como dificuldades na identificação e na qualificação da população beneficiária por esta rede. Demonstrou também o quão ainda é superficial o conhecimento sobre os serviços e a relevância deste, especialmente na atenção básica, porta de entrada para qualquer serviço do SUS.

Acredita-se que esta situação é decorrente da grande rotatividade de funcionários nesse nível, o que tornam necessárias ações de educação permanente, sendo estas o principal caminho para a consolidação da rede de cuidados à pessoa com deficiência.

Além disso, programas de consultorias como os blogs dos serviços, e-mail e telessaúde, mantidos pelo projeto de extensão, são uma forma de atender prontamente aos questionamentos da atenção básica, conservando assim a comunicação entre os níveis.

Em relação ao projeto de extensão, este permitiu estabelecer um vínculo maior entre os diferentes níveis de atenção à saúde da pessoa com deficiência e possibilitou aos acadêmicos uma vivência prática apoiada na construção de redes de apoio à pessoa com deficiência e ao fortalecimento da integração ensino-serviço.

Para os próximos anos, o projeto visa ampliar as capacitações a outros municípios da AMFRI, além de voltar aos municípios em que as capacitações já foram realizadas com outras temáticas relacionadas à deficiência, conforme a demanda de cada território. Para isso a elaboração do menu de capacitações organiza o que pode ser oferecido pelo projeto, corroborando com o que foi apresentado em uma reunião do grupo condutor da pessoa com deficiência, em que participam os municípios.

Por fim, sugere-se como mecanismo de controle e avaliação contínua das ações do projeto o acompanhamento do número e da qualidade dos encaminhamentos do município em questão aos serviços oferecidos pela rede nos próximos anos.

\section{REFERÊNCIAS}

ALVES, E. D. Política de Educação e Desenvolvimento para o SUS - Caminhos para a Educação Permanente em Saúde. Revista Eletrônica Gestão \& Saúde, Brasília, v.1, n.1, p.42-47, 2017.

AYRES, J. R. C. M. Extensão universitária: aprender fazendo, fazer aprendendo. Revista de Medicina, São Paulo, v.94, n.2, p.75-80, 2015.

BRASIL, Ministério da Saúde. Política de educação e desenvolvimento para o SUS: caminhos para a Educação Permanente em saúde. Brasília, 2004. 
BRASIL. Ministério da Saúde. Decreto n ${ }^{\circ}$ 5296/04 de 02 de dezembro de 2004: Regulamenta as Leis nos 10.048 , de 8 de novembro de 2000, que dá prioridade de atendimento às pessoas que especifica, e 10.098, de 19 de dezembro de 2000 que estabelece normas gerais e critérios básicos para a promoção da acessibilidade das pessoas portadoras de deficiência ou com mobilidade reduzida, e dá outras providências, 2004.

BRASIL. Ministério da Saúde. Portaria GM/MS no 1.996, de 20 de agosto de 2007: Política Nacional de Educação Permanente em Saúde, 2007.

BRASIL. Ministério da Saúde. Política nacional de saúde da pessoa portadora de deficiência. 2011.

BRASIL. Ministério da Saúde. Portaria no 793/12 de 24 de abril de 2012: institui a rede de cuidados à pessoa com deficiência no âmbito do Sistema Único de Saúde, 2012.

FERREIRA, S. P.; CURRY, L. K. P. Gestão Integrada com a Utilização do Sistema Informatizado. 2013. Disponível em: <http://www.portalcatalao.com/painel_clientes/cesuc/painel/arquivos/upload/downlo ads/2f8b13edoc6eac89594fbb5752foa684.pdf >. Acesso em: 23 mar. 2018.

GODOY, S. C. B; GUIMARÃES, E. M. P; ASSIS, D. S. S. Avaliação da capacitação dos enfermeiros em unidades básicas de saúde por meio da telenfermagem. Escola Anna Nery Revista de Enfermagem, v.18, n.1, p.148-155, jan./mar. 2014.

IBGE. Cartilha do Censo 2010, Brasília, 2012. Disponível em: < http://www.pessoacomdeficiencia. gov.br/app/sites/default/files/publicacoes/cartilha-censo-2010-pessoas-com-deficienciareduzido.pdf $>$. Acesso em: 26 mar. 2018.

LEMOS, C. L.S. Educação Permanente em Saúde no Brasil: educação ou gerenciamento permanente? Ciência e Saúde Coletiva, Rio de Janeiro, v.21, n.3, p. 913-922, mar. 2016.

MICCAS, F. L.; BATISTA, S. H. S. S. Educação permanente em saúde: metassíntese. Revista de Saúde Pública, São Paulo, v.48, n.1, p.170-185, fev. 2014.

PEREIRA, J. S.; MACHADO, W. C. A. Referência e contrarreferência entre os serviços de reabilitação física da pessoa com deficiência: a (des)articulação na microrregião Centro-Sul Fluminense, Rio de Janeiro, Brasil. Physis - Revista de Saúde Coletiva, Rio de Janeiro, v.26, n.3, p.1033-1051, 2016.

SANTOS, R. C. A.; MIRANDA, F. A. N. Articulação Ensino-Serviço na Perspectiva dos Profissionais de Saúde da Família. Revista de APS, v.19, n.1, 2016.

SILVA, L. S. G; GONÇALVES, C. C. O.; SOARES, V. M. N. National Policy on Health Care Hearing: an evaluative study from covering services and diagnostic procedures. CoDAS, v.26, n.3, p.241-247, 2014.

TOCCI, A. S. S; COSTA, E. C. N. F. A gestão em saúde após a política nacional de humanização no Sistema Único de Saúde - SUS. Revista Uningá, Maringá, v.40, p.197-206, abr./jun. 2014.

URSINE, B. L.; PEREIRA, É. L.; CARNEIRO, F. F. Saúde da pessoa com deficiência que vive no campo: o que dizem os trabalhadores da Atenção Básica? Interface - Comunicação, Saúde, Educação, Botucatu, v.22, n.64, p.109-120, jan./mar. 2018.

UNIVALI, Universidade do Vale do Itajaí. Centro Especializado em Reabilitação Física e Intelectual. 2015. Disponível em: http://www.univali.br/. Acesso em: 20 de novembro de 2015. 